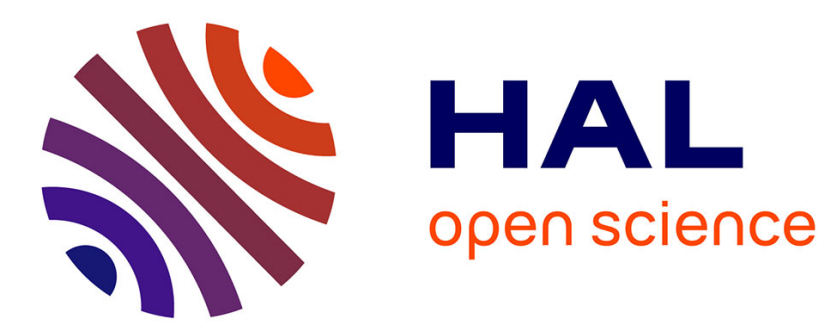

\title{
Les fouilles de Gergovie
}

Albert Grenier

\section{To cite this version:}

Albert Grenier. Les fouilles de Gergovie. Gallia - Fouilles et monuments archéologiques en France métropolitaine, 1943, 1 (2), pp.71-82. 10.3406/galia.1943.1968 . hal-01920352

\section{HAL Id: hal-01920352 \\ https://hal.science/hal-01920352}

Submitted on 10 Mar 2020

HAL is a multi-disciplinary open access archive for the deposit and dissemination of scientific research documents, whether they are published or not. The documents may come from teaching and research institutions in France or abroad, or from public or private research centers.
L'archive ouverte pluridisciplinaire HAL, est destinée au dépôt et à la diffusion de documents scientifiques de niveau recherche, publiés ou non, émanant des établissements d'enseignement et de recherche français ou étrangers, des laboratoires publics ou privés.

\section{(이) $\$$}

Distributed under a Creative Commons Attribution - NonCommercial - NoDerivatives 44.0 


\section{LES FOUILLES DE GERGOVIE}

\section{OPPIDUM DE GERGOVIE}

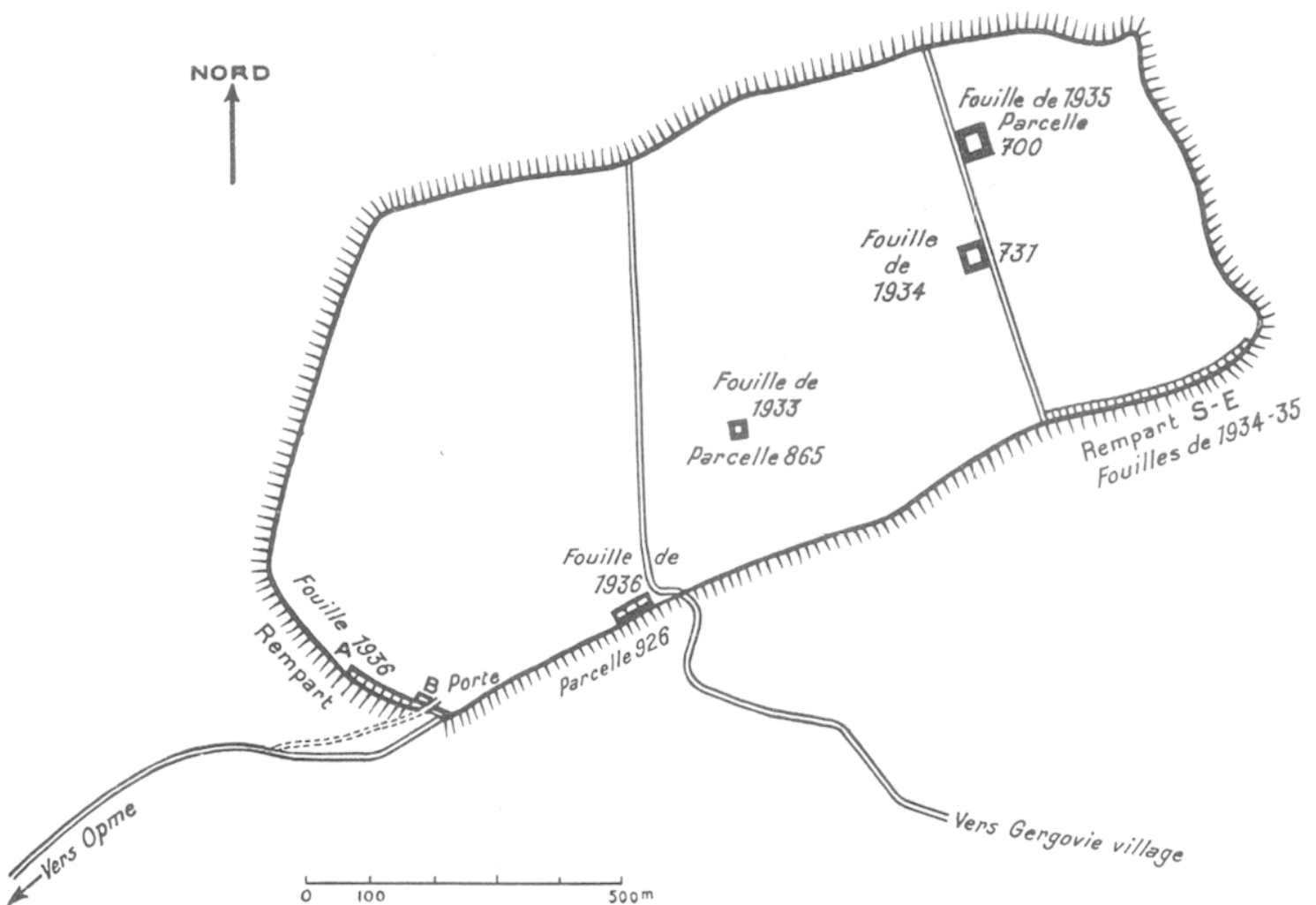

Plan I. Plan schématique de l'oppidum donnant l'emplacement des principaux points de fouilles.

Les fouilles de Gergovie, dont nous publions ci-dessous le compte rendu détaillé, ont des antécédents que l'on ne saurait manquer de rappeler.

En 1932 s'est constitué à Clermont-Ferrand un comité Pro Gergovia, en vue de donner une impulsion plus active et méthodique aux recherches individuelles qui n'ont jamais cessé sur le plateau. Les premiers fonds recueillis avaient permis de subvenir en 1934 aux frais d'une brève campagne de fouilles qui aboutit à dégager sur le front sud du plateau 

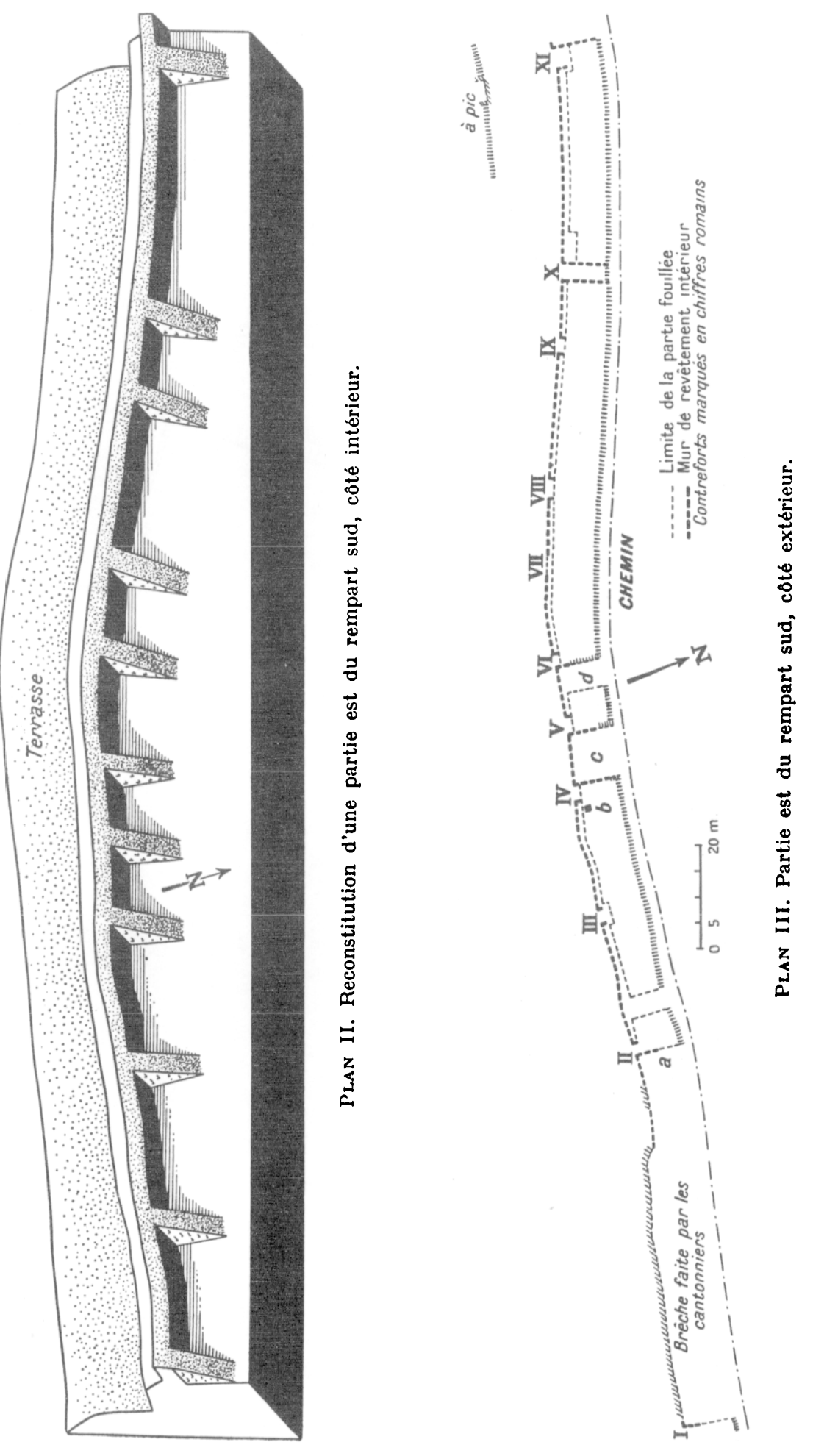

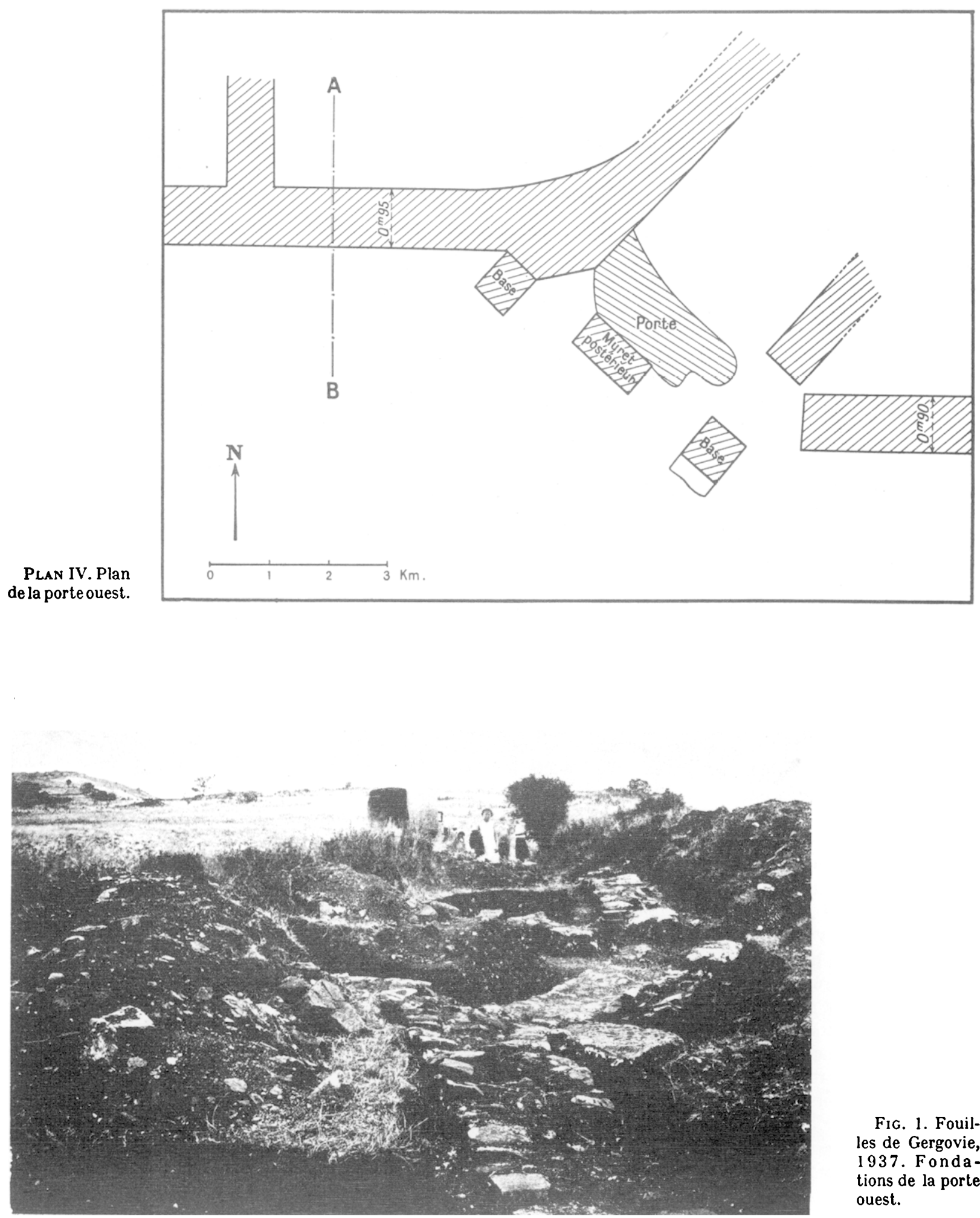

FIg. 1. Fouilles de Gergovie, 1937. Fondations de la porte ouest. 
un système défensif composé, à la base, d'une paroi de basalte à peu près verticale et, au-dessus, d'un agger, construit à pierre sèche.

A l'intérieur de cette enceinte furent mises au jour diverses substructions qui, d'après les trouvailles, semblent du début de l'époque romaine : sols bétonnés, fragments de mosaïque blanche et noire, enduits peints, monnaies gauloises, céramique de La Tène et fragments de terre sigillée, monnaies de Trajan, etc. Les fouilles étaient dirigées par M. E. Desforges, de Clermont-Ferrand. Deux archéologues anglais, Mme Brogan et M. Hawkes, y ont apporté une collaboration efficace. Un bref rapport a paru dans la Revue archéologique, 1935, I, p. 220-230 (cf. Rev. Ét. anc., 1936, p. 48). Un autre compte rendu était donné peu après dans la revue anglaise Antiquity, 1936, p. 210-217 (cf. Rev. Ét. anc., 1937, p. 38). Une étude plus détaillée de ces fouilles et de celles des années suivantes a dû paraitre, depuis la guerre, sous la signature des trois fouilleurs, dans une revue anglaise, dont nous n'avons naturellement pas eu communication; nous en donnerons plus tard la référence et l'analyse.

Depuis 1935, de modestes subventions de la Direction des Beaux-Arts aidèrent le comité Pro Gergovia à poursuivre ces recherches (v. le plan I). Elles ont porté sur le rempart et sur quelques points du plateau.

I. Le rempart. En 1935 a été dégagée une partie du mur sud-est. Le système défensif comportait de ce côté un premier rempart séparé par une terrasse d'une deuxième fortification constituée par un tertre de pierres et de terre, protégé lui-même par un escarpement taillé dans le basalte.

Dans ce tertre étaient incluses d'énigmatiques constructions, sortes de cellules en pierre sèche sur trois côtés, le quatrième étant ouvert sur l'intérieur du plateau. (Plan II et vue cavalière schématique). Il ne s'agissait pas, comme on l'avait cru tout d'abord, d'anciennes habitations adossées au rempart : ces cavités faisaient corps, au contraire, avec le rempart. En effet, à l'intérieur du tertre fut dégagé le parement intérieur d'un mur enseveli sous les matériaux d'épierrage. Construit en pierre sèche, sa hauteur actuelle est, en moyenne, de $1 \mathrm{~m}$. 50. Perpendiculairement à ce mur et sur sa face interne, des murets, larges de 1 à 2 mètres, longs de 5 mètres, ont été découverts à des distances irrégulières de 4 à 12 mètres. Ce mur avec ses contreforts a été mis au jour sur une longueur de 100 mètres (plan III); la construction en a été vérifiée sur d'autres points jusqu'à l'extrémité ouest du plateau. Elle a été retrouvée partout identique.

En 1936 et 1937, les recherches ont été portées vers l'ouest du plateau où les traces de la fortification subsistent sous la forme d'un tertre gazonné d'une longueur d'environ 500 metres. Un tronçon de 150 mètres a été dégagé. On a pu y constater que le rempart était constitué par un mur épais de $2 \mathrm{~m}$. 20 formé de deux parements en pierre sèche, l'intervalle étant comblé par de la pierraille mélangée de terre. En B, le mur était coupé par une brèche donnant passage à un ancien chemin (plan I). Comme l'avait constaté l'agent-voyer Auclerc en 1861, cette brèche conservait la trace d'une ancienne porte.

La porte avait une largeur de 3 mètres (plan IV, fig. 1 et 2). Deux fortes bases carrées en pierre devaient en supporter les deux montants. Une salle rectangulaire de $4 \mathrm{~m}$. 50 sur $4 \mathrm{~m}$. 40, dont un des murs faisait corps avec le rempart, devait servir de corps de garde. Les murs en sont construits au mortier et ont une moyenne de $0 \mathrm{~m}$. 90 d'épaisseur. Deux autres salles de même construction ont été reconnues entre le rempart voisin de la porte et le plateau. Les trouvailles sont demeurées peu abondantes : quelques fibules de bronze et de fer du type La Tène III, quelques monnaies d'Epadnactus, en bronze, au type du guerrier à pied, beaucoup de clous et de scories de fer. Ja céramique est en majeure partie du type du Beuvray, mais en un point ont été trouvés quelques tessons d'Arezzo associés à une belle monnaie de Nimes. Des sondages sous les fondations des murs ont donné des fragments d'une céramique de pâte et de lustre identiques à celle qui avait été trouvée 


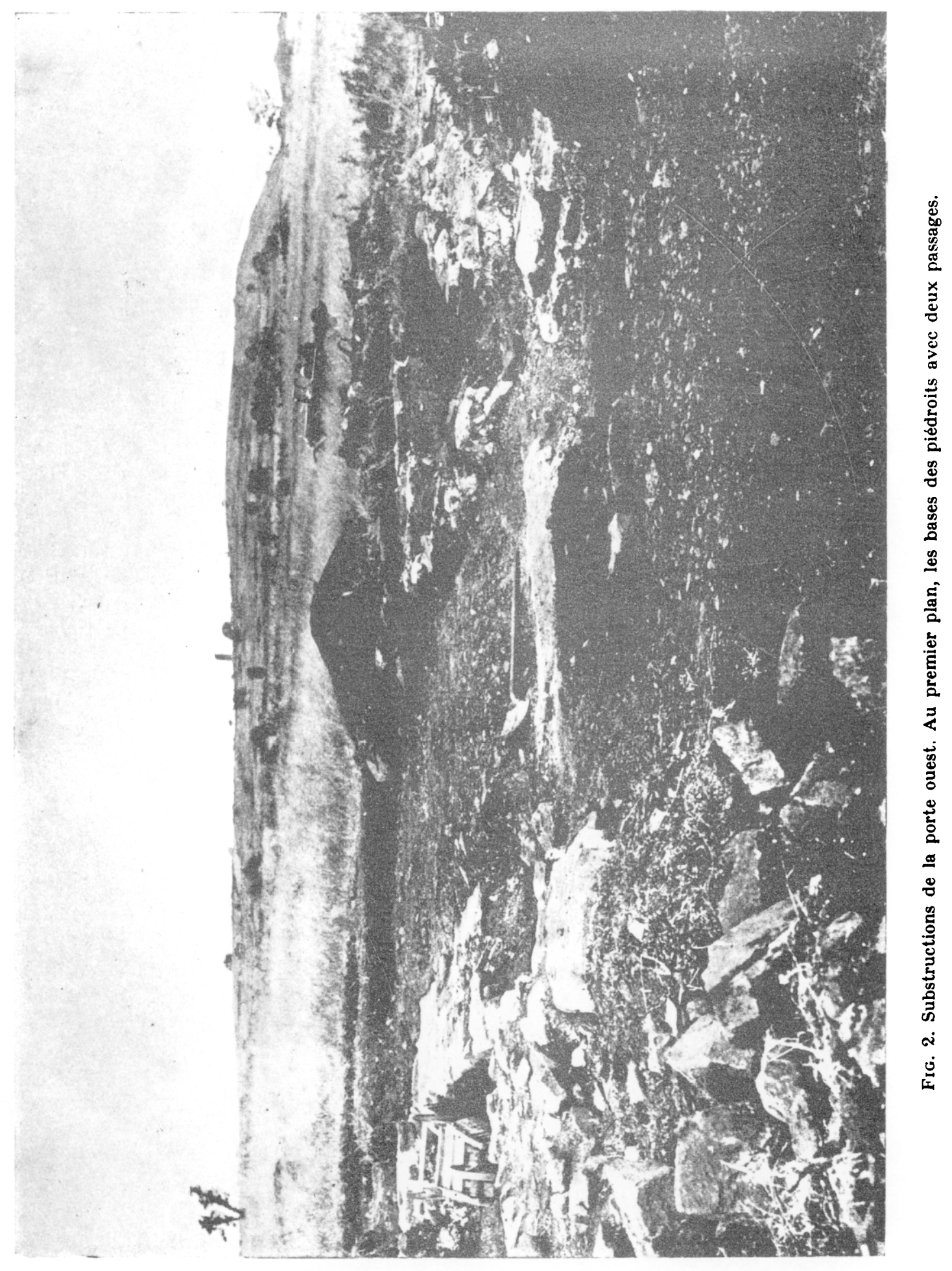




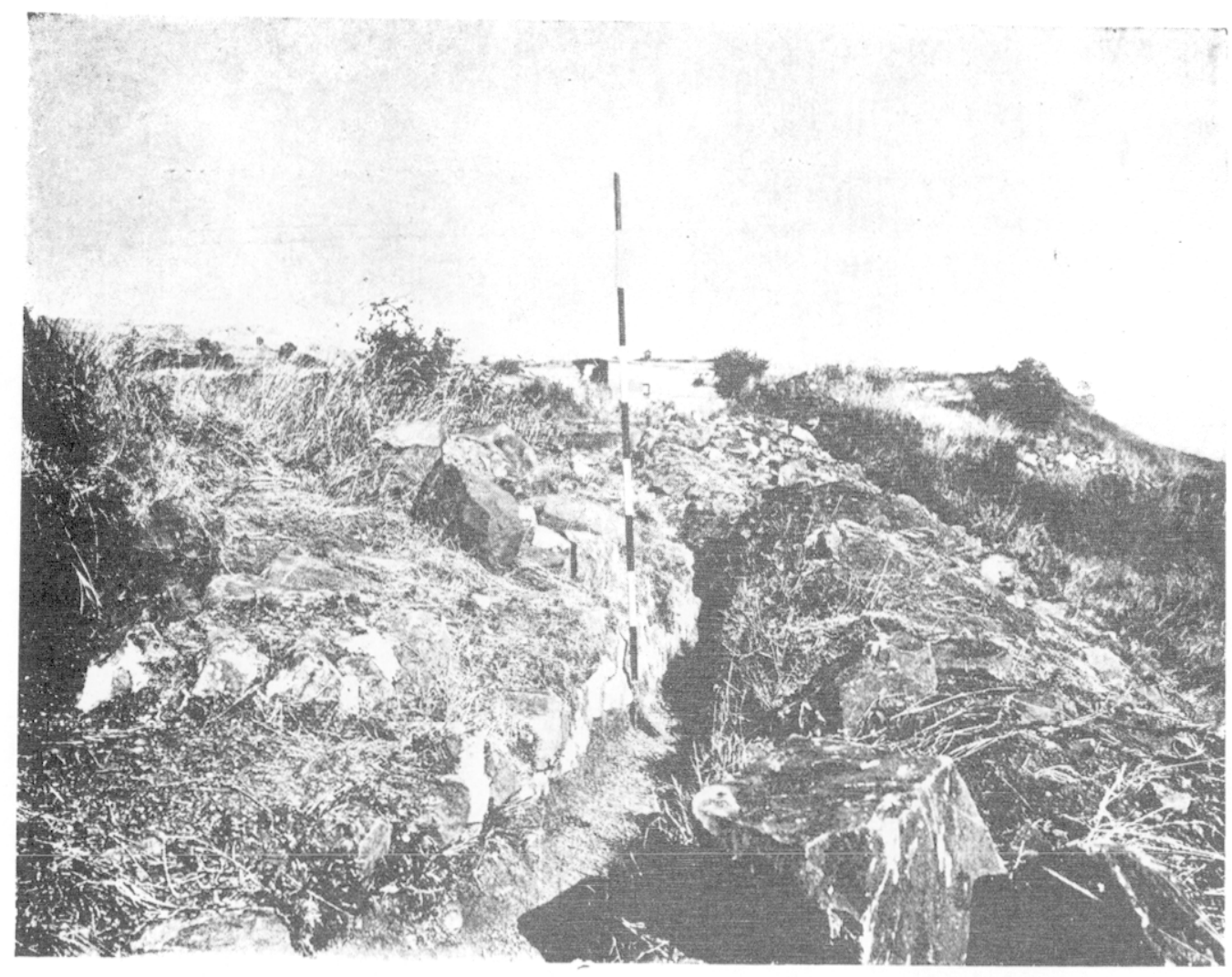

Fig. 3. Parcelle 926. Dégagement du rempart (mur extérieur) ouest près de la porte.

Fig. 4. Mur dégagé du rempart ouest, parement intérieur.

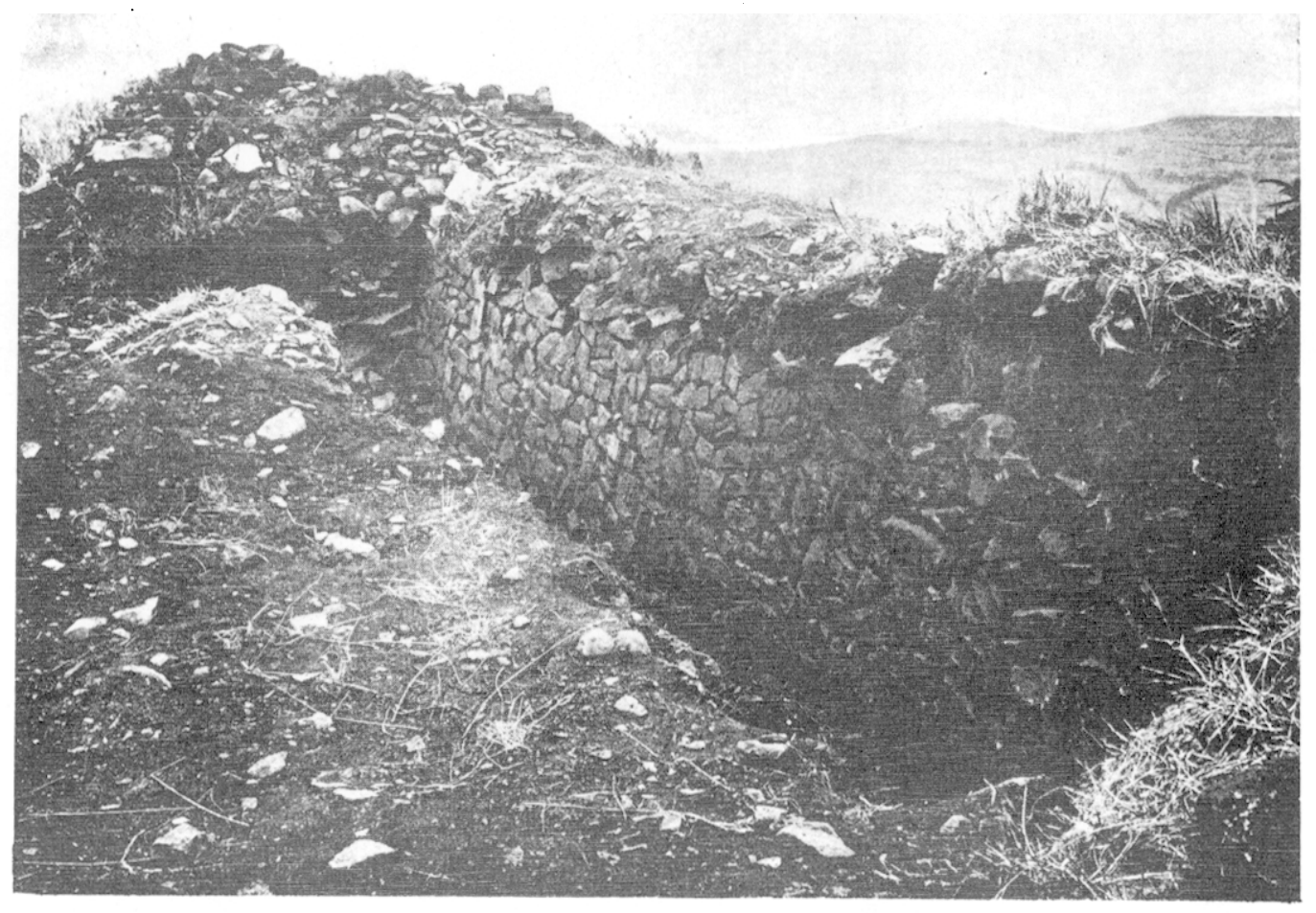


sous la base des remparts du sud-est du plateau, céramique qui semble hallstattienne. De ces recherches, les fouilleurs tiraient les conclusions suivantes :

$1^{\circ}$ Le rebord ouest du plateau de Gergovie, comme son extrémité est, a été occupé au début du premier âge du Fer;

$2^{\circ}$ Sur ces vestiges d'occupation, un mur en pierre sèche, véritable fortification du plateau, a été élevé à une date indéterminée de l'époque indépendante ;

$3^{\circ}$ Postérieurement à l'établissement de ce mur, une porte avec bâtiments de garde a été construite au mortier de chaux, mais aver une technique encore très rudimentaire;

$4^{\circ}$ Le chemin d'accès au plateau, venant de l'ouest, se dirigeait vers le centre de l'oppidum, selon la trace

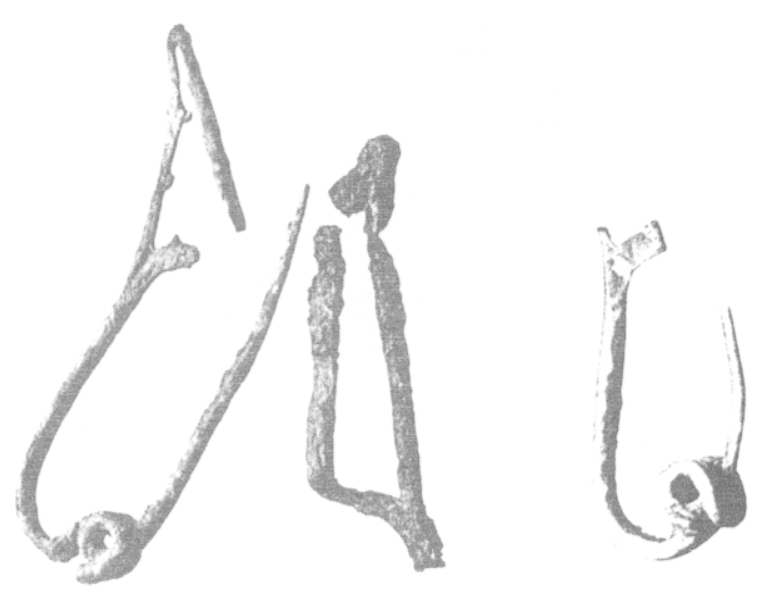

Fig. 5. Gergovie. Parcelle 731.

Fibules trouvees dans le puits perdu. d'une voie indiquée au xvine siècle sur le plan de Caylus et qui ne fut détruite que lors du morcellement des terrains opéré sous la Révolution.

II. A l'intérieur du rempart, des fouilles, faites un peu au hasard des ententes avec les propriétaires, ont mis au jour les restes d'habitations privées ou de monuments publics.

Parcelle 731 (1934) : construction de pierre et de mortier avec sol bétonné des fragments de mosaïque blanche et'noire, enduits peints, une monnaie gauloise, une conduite d'eau en tuiles à rebords et un puisard rempli de débris de toute sorte (fig. 5) et surtout de tessons en majeure partie de céramique grise ou noire du type du Beuvray, avec décoration en feuilles de fougère, damiers, yeux, lignes ondées. Quelques tessons sont recouverts

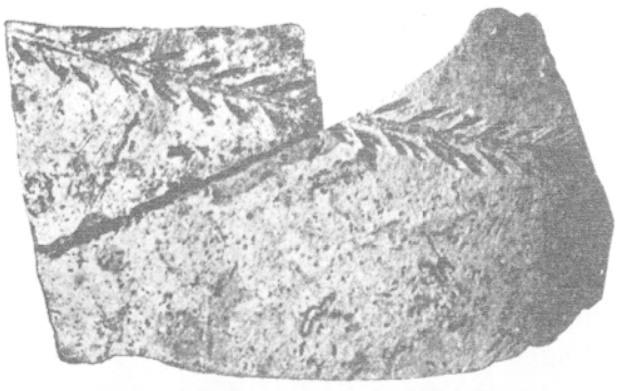

Fig. 6. Gergovie. Parcelle 731. Céramique à engobe blanc et décor, trouvée au fonds du puits perdu.

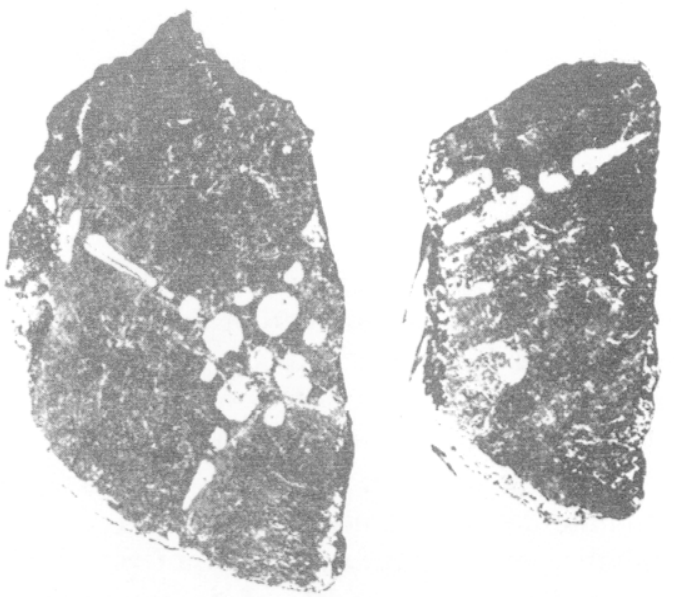

Fig. 7. Gergovie. Parcelle 731. Enduit peint en rouge avec décor blanc. 
d'un engobe blane très fragile orné de feuillage (fig. 6 et 7). Quelques fragments de terre sigillée et de gobelets d'Acco.

Parcelle 700 et avoisinantes (1935) : substructions d'un édifice avec un portique long de 50 mètres et comprenant 14 colonnes, dont quelques bases subsistaient encore (fig. 8). Les colonnes étaient composées de briques en quart de cylindre, unies par de l'argile. Le portique formait la façade ouest d'un bâtiment important, probablement édifice public.

Le bâtiment central paraît avoir été constitué par deux salles incluses l'une dans l'autre et dont le sol était revêtu d'un pavage en mortier à tuileaux; dans la

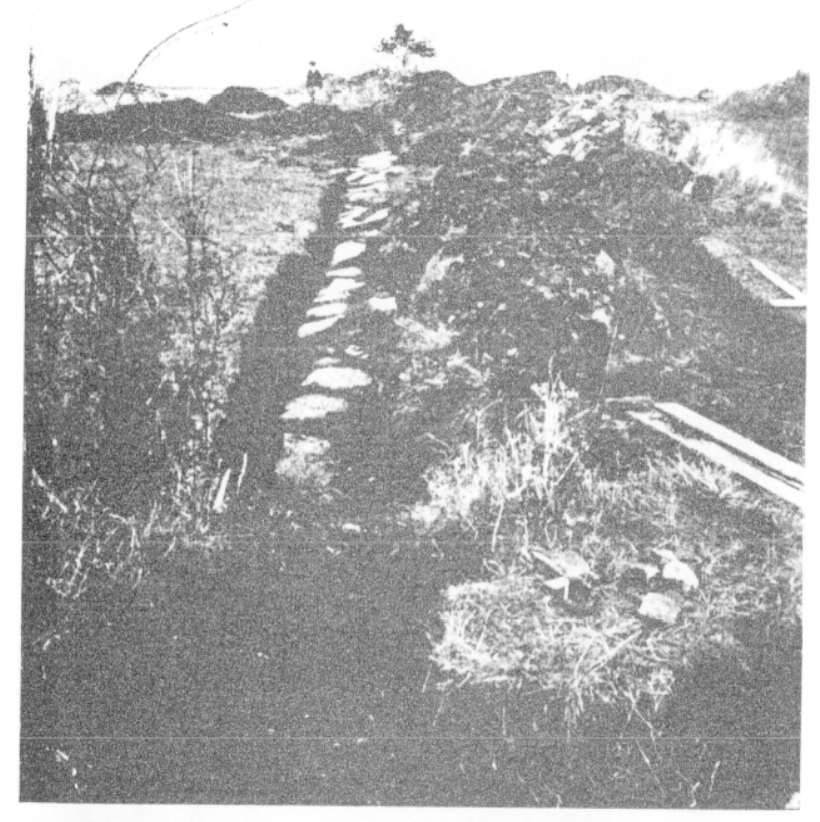

FIG. 8. Substructions d'un édifice :

bases de 14 colonnes, sur 50 mètres de long. petite salle de gros dés de marbre blanc étaient encastrés çà et là dans les tuileaux.

Les trouvailles ont été sensiblement les mêmes que dans la fouille précédente : grande quantité de tessons du type Beuvray, fragments de gobelets d'Acco; deux tessons à glaçure jaune paraissant provenir des ateliers de Saint-Remy-en-Rollat.

Les murs sont montés en basalte du plateau et très souvent sans chaux. Ils sont peu épais : $0 \mathrm{~m}$. 50 en moyenne à la base ; pour le bâtiment central, l'épaisseur varie entre $0 \mathrm{~m} .60$ et $0 \mathrm{~m} .70$.

Parcelles 926-927 (1936) (plan V). Les tranchées creusées en ce lieu ont permis de déterminer en partie le plan d'un bâtiment appuyé (fig. 9) contre le rempart et compartimenté en pièces de dimensions très réduites, puisque la plus grande ne mesure que $4 \mathrm{~m}$. 25 sur $4 \mathrm{~m}$. 50. Cette pièce est plus ancienne que l'ensemble de l'édifice tel qu'il existait lors de sa destruction finale. Le niveau inférieur s'en trouve, en effet, à $2 \mathrm{~m}$. 40 au-dessous du sol du plateau; les parois sont établies grossièrement en pierre sèche. Une autre construction lui a été superposée à $2 \mathrm{~m}$. 20 de profondeur, puis une troisième, d'un plan tout autre et dont le niveau se trouve actuellement à $1 \mathrm{~m}$. 60 de profondeur.

L'étage inférieur n'a fourni que des poteries grossières ; l'étage intermédiaire ( $2 \mathrm{~m}$. 20) a donné de la céramique grise de La Tène III mélangée à des débris d'amphore de type italique, ainsi qu'un tesson de La Graufesenque avec la signature CELER. De nombreuses traces d'incendie, cendres et débris de poutres calcinées, sont encore visibles.

L'étage supérieur a fourni surtout de la céramique à engobe blanc du ${ }_{i}{ }^{\text {er }}$ siècle de notre ère. Les murs en sont construits en excellent mortier. Un certain nombre de monnaies, toutes gauloises, ont été trouvées çà et là au cours des travaux organisés sur ce point. 


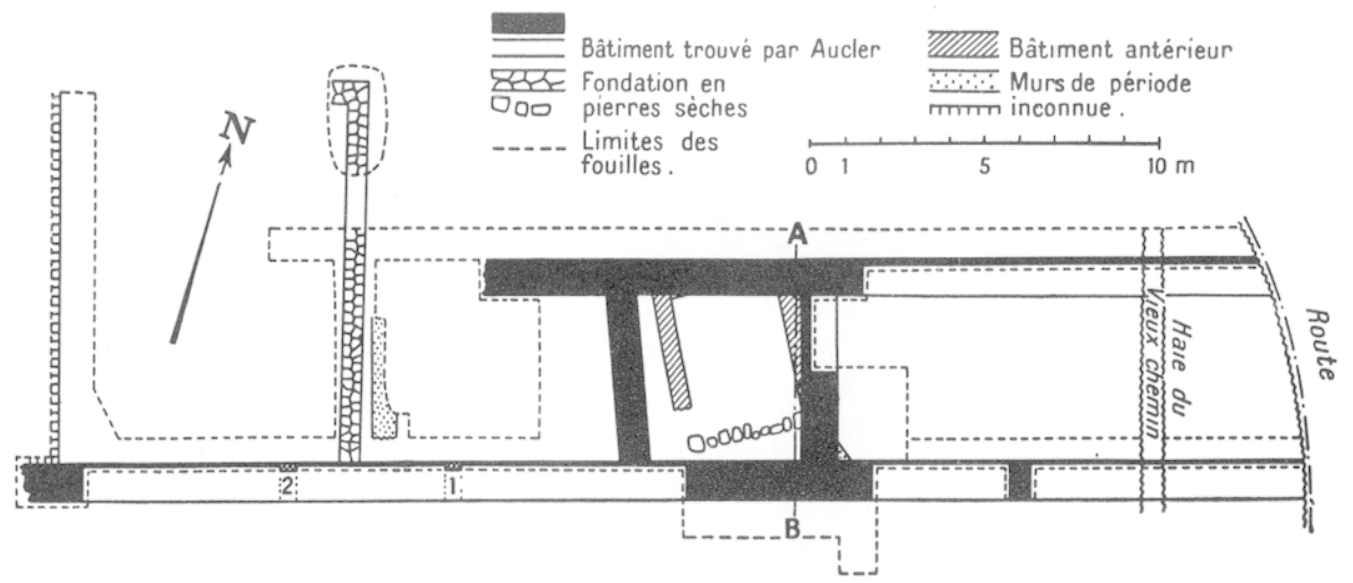

Plan V. Parcelle 926. Restes d'un bâtiment appuyć contre le rempart.

Enceinte à deux temples (1937).

La reprise des travaux dans les parcelles $700-701$ a permis d'y reconnaftre deux temples, du type des sanctuaires carrés bien connus dans les provinces celtiques de l'Empire romain ; ils étaient entourés d'un péribole commun (plan VII et fig. 10).

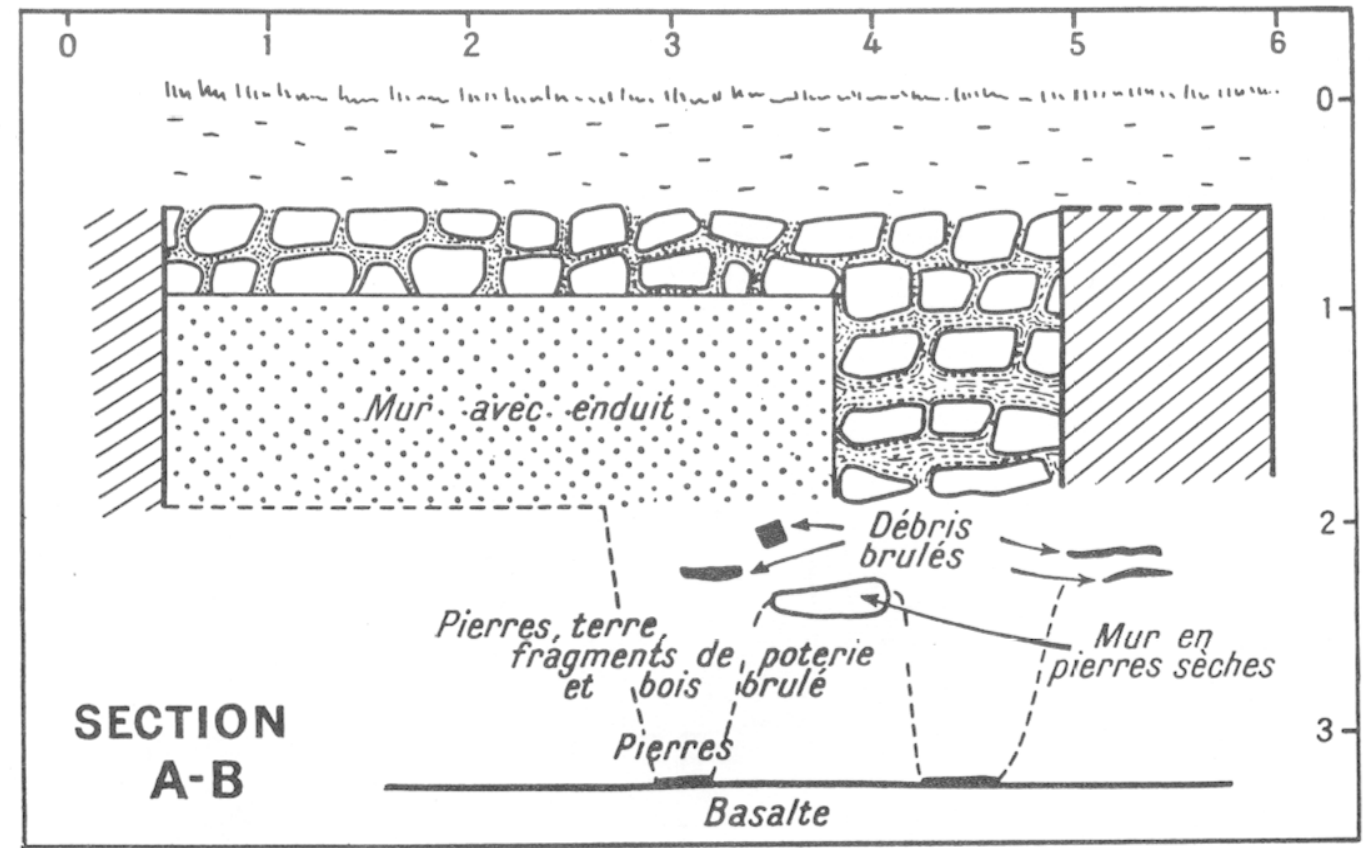

Plan VI. Section A-B du plan V. 


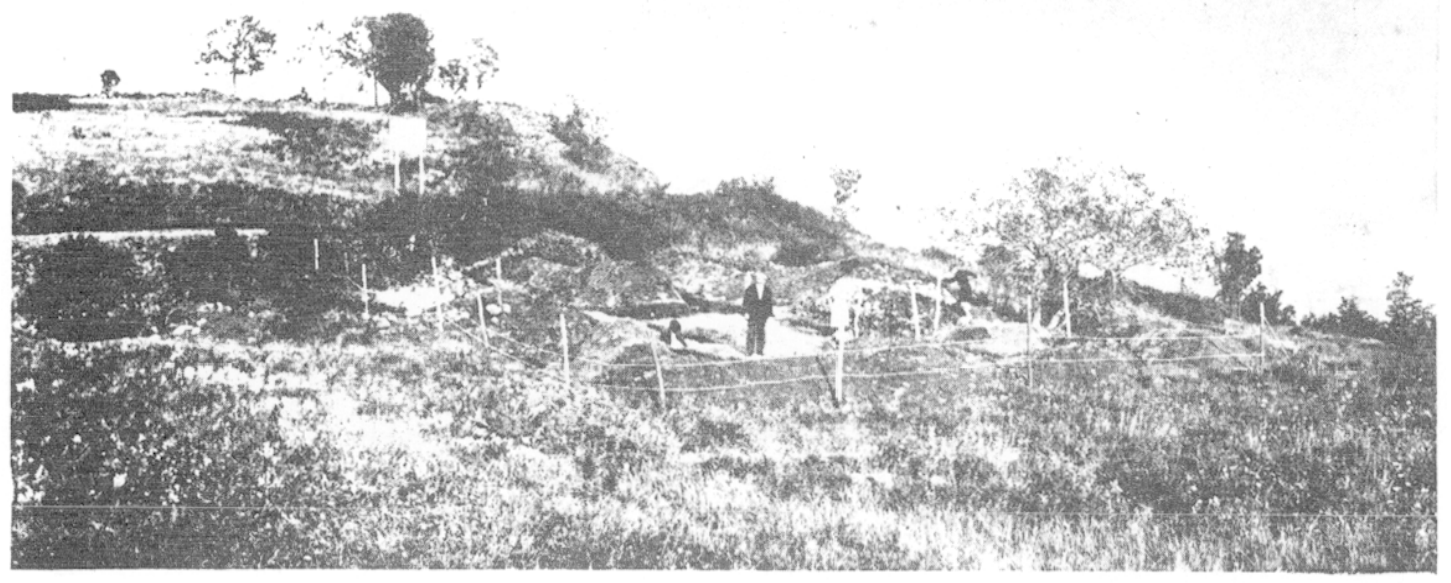

Fic. 9. Ensemble des fouilles de la parcelle 926 .

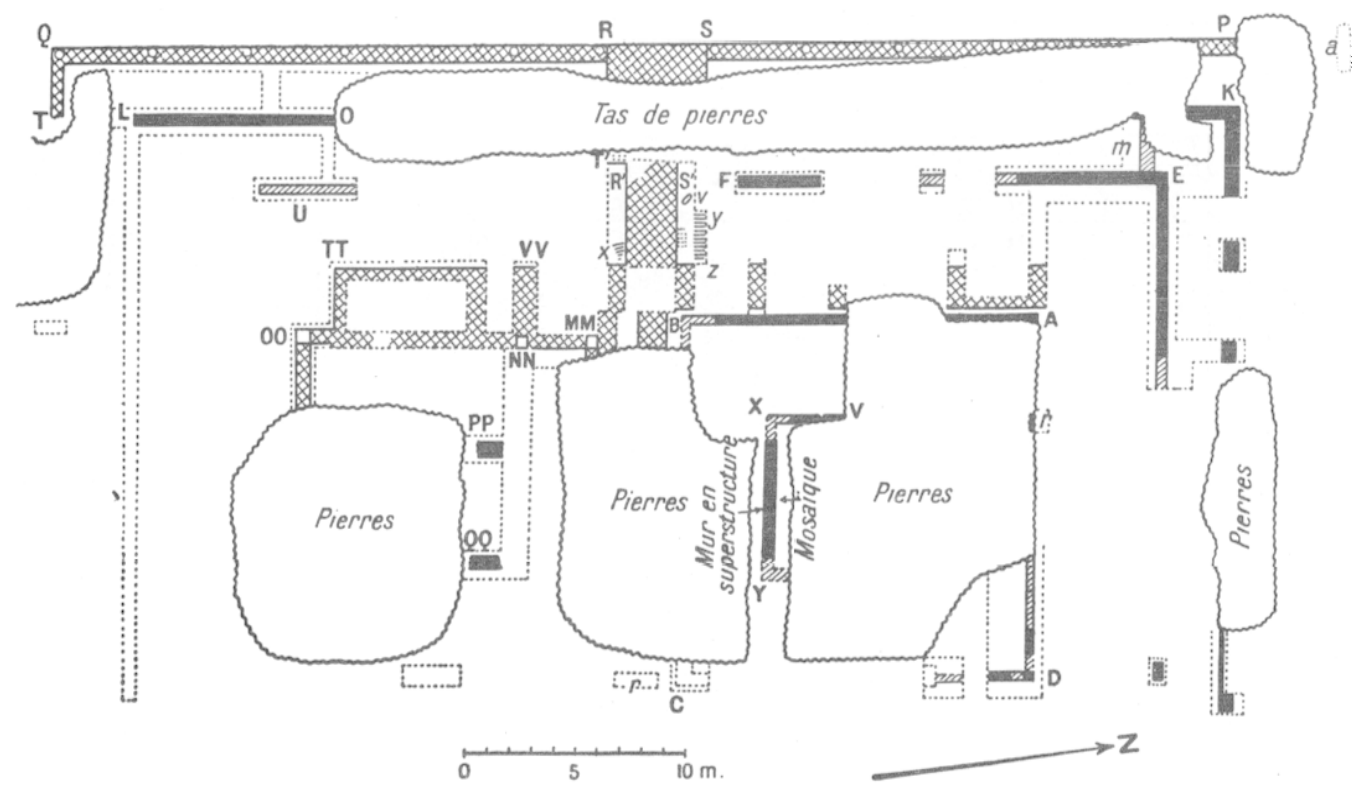

Plan VII. Parcelles 700-701. Substructions de deux temples entourés d'un peribole commun. 


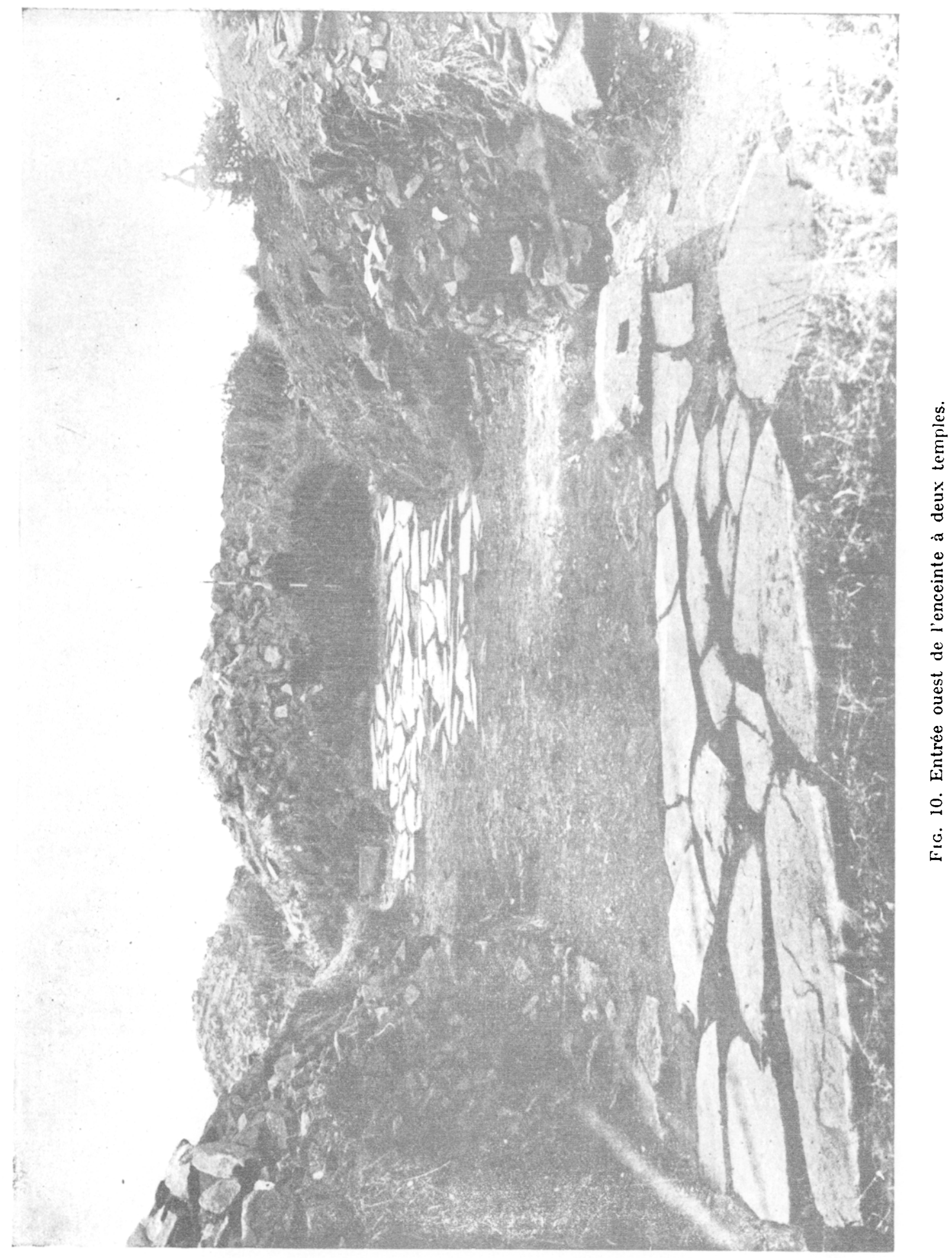




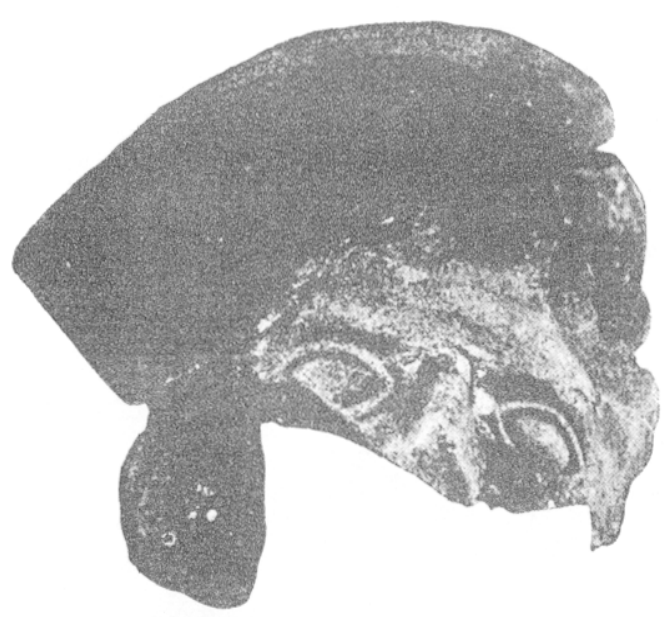

Frg. 11. Antéfixe en terre cuite trouvée sous le béton de la cella du temple sud.
Le temple nord se compose de deux carrés concentriques. Les murs du carré extérieur sont moins épais que ceux de la cella : $0 \mathrm{~m} .40$ à $0 \mathrm{~m}$. 50 contre $0 \mathrm{~m} .90$ pour les fondations et $0 \mathrm{~m} .60$ pour le mur luimême. Le sol de la cella était recouvert d'un béton composé de débris d'amphores et menus morceaux de céramique diverse, noyé dans du ciment. La porte d'entrée de la cella se trouvait au milieu du côté est.

Le temple sud est également de forme carrée ; la cella en était entourée, non d'un autre mur, mais d'une colonnade. Le sol de la cella et de la colonnade était constitué d'un béton peu épais et de mauvaise qualité. Ce temple a beaucoup souffert; les murs et le sol de la partie nord ont entièrement disparu. En fouillant en cet endroit jusqu'au basalte, on a retrouvé une monnaie de la République T. POMP. SEX. F ; avers : un profil ; revers : taureau, ainsi qu'un fragment d'antéfixe en terre cuite provenant évidemment d'un bátiment antérieur (fig. 11). La couche de terre fouillée sous le béton de la cella a donné quelques tessons de céramique primitive de type hallstattien.

Les fouilles en ce point ne sont qu'amorcées, mais ne pourront être continuées que lorsque le terrain aura été débarrassé des amas de pierres qui l'encombrent (1).

Les objets trouvés ont été déposés sur place à l'auberge de la - Hutte » sur le plateau, où une salle a été mise à la disposition de l'Académie de Clermont pour l'aménagement provisoire d'un Musée gergovien.

En 1941, le comité Pro Gergovia, reconstitué, s'est adjoint de nouveaux membres, avec l'aide desquels il a repris les fouilles.

A. G.

(Albert Grenier)

(1) Ces renseignements sont extraits des rapports adressés par MM. P. Fournier ef $E$. Desforges à la Direction des Beaux-Arts. Ces rapports s'arrêtent en 1937.

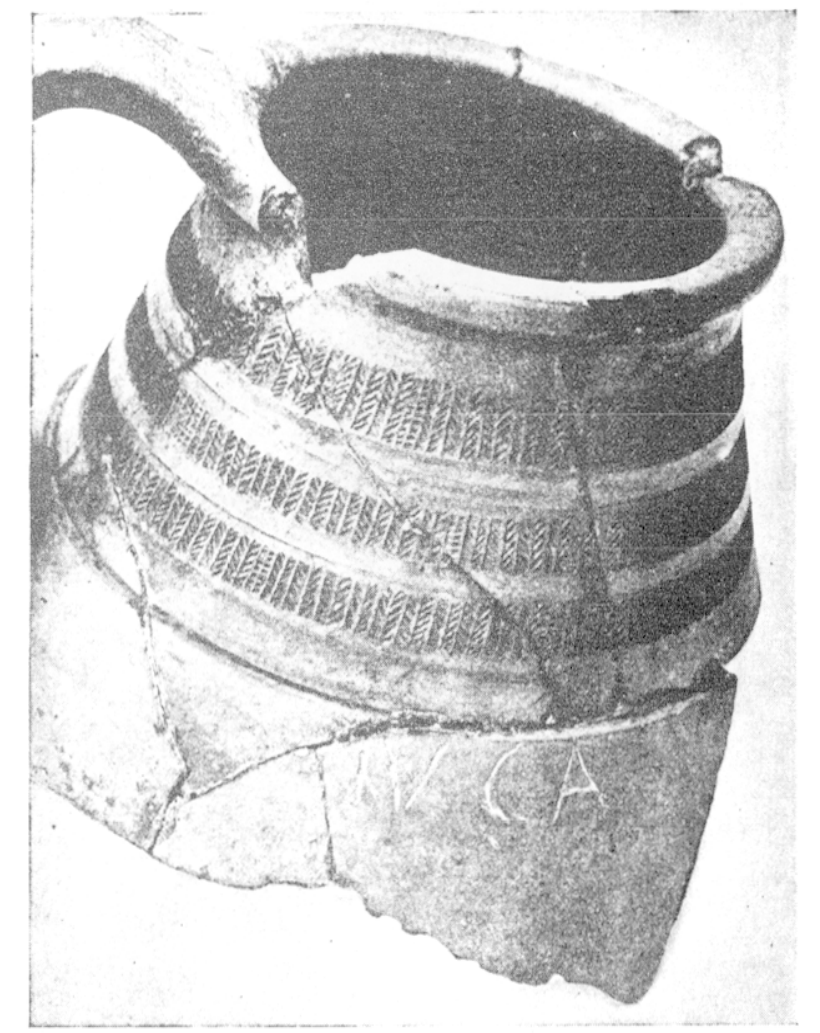

FIG. 12. Céramique grise à décor beuvraysien (enceinte à deux temples). 